\title{
Geranylgeranylacetone attenuates hepatic fibrosis by increasing the expression of heat shock protein 70
}

\author{
WEI HE* , YUN ZHUANG ${ }^{*}$, LIANGZHI WANG, LEI QI, BINFANG CHEN, \\ MEI WANG, DONG SHAO and JIANPING CHEN \\ Department of Gastroenterology, The Third Affiliated Hospital of Soochow University, \\ Changzhou, Jiangsu 213003, P.R. China
}

Received August 30, 2014; Accepted June 3, 2015

DOI: $10.3892 / \mathrm{mmr} .2015 .4069$

\begin{abstract}
Increasing evidence has demonstrated that the heat shock protein 70 (HSP70) gene may be closely associated with tissue fibrosis; however, the association between HSP70 and liver fibrosis remains to be fully elucidated. The present study hypothesized that geranylgeranylacetone (GGA) exerts beneficial effects on liver fibrosis though upregulation of the expression of HSP70. Liver fibrosis was induced in rats using carbon tetrachloride $\left(\mathrm{CCl}_{4}\right)$. The rats were subsequently divided into three groups: Control group, $\mathrm{CCl}_{4}$ model group and $\mathrm{CCl}_{4}$ model + GGA group. Liver fibrosis in the rats was evaluated using hematoxylin and eosin staining, Masson's trichrome staining and Sirius red staining. The levels of serum alanine aminotransferase, aspartate aminotransferase and total bilirubin were determined using an automated biochemistry analyzer. The levels of total hepatic hydroxyproline were also determined. The expression levels of $\alpha$-smooth muscle actin ( $\alpha$-SMA) and transforming growth factor- $\beta 1$ (TGF- $\beta 1)$ were determined using immunofluorescence staining and western blotting, and the protein expression levels of HSP70 were determined using western blotting. The $\mathrm{CCl}_{4}$-induced rats exhibited liver fibrosis, increased hydroxyproline content, impaired liver function, upregulated expression levels of the $\alpha$-SMA and TGF- $\beta 1$ pro-fibrogenic proteins, and increased expression of HSP70, compared with the control group. These changes were attenuated by treatment with GGA. These results demonstrated that GGA exerted beneficial effects in $\mathrm{CCl}_{4}$-induced liver fibrosis via upregulating the expression of HSP70.
\end{abstract}

Correspondence to: Professor Jianping Chen, Department of Gastroenterology, The Third Affiliated Hospital of Soochow University, 185 Juqian Road, Changzhou, Jiangsu 213003, P.R. China E-mail: chenjianping7688@163.com

*Contributed equally

Key words: geranylgeranylacetone, liver fibrosis, heat shock protein 70

\section{Introduction}

Liver fibrosis is the common response to chronic liver injury, which is characterized by accumulation of extracellular matrix proteins and can result in the loss of liver function and disruption of liver tissue structure, ultimately leading to irreversible cirrhosis $(1,2)$. Due to the lack of effective therapies, cirrhosis-associated mortality has been increasing annually. It is increasingly considered that the most effective method to prevent and treat cirrhosis is to inhibit the development of hepatic fibrosis (3).

Heat shock proteins (HSPs) are a family of polypeptide proteins consisting of several members, which perform important housekeeping functions and are named according to their molecular weight, including HSP105/110, Hsp90, HSP70, HSP60, HSP40, and other low molecular weight HSPs $(4,5)$. HSP70 chaperones and their co-chaperones comprise a set of abundant cellular machines, which assist a variety of protein folding processes in almost all cellular compartments (6). They provide an essential action through preventing aggregation and assisting refolding of misfolded proteins (7). By regulating cell apoptosis and autophagy, reducing oxidative stress and resisting inflammation, HSP70 protects the body against acute and subacute injury (8). Previous studies have demonstrated that the HSP70 gene may be closely associated with tissue fibrosis: Firstly, high expression levels of HSP70 have been detected in the livers of patients with cirrhosis and of animals with hepatic fibrosis (9); secondly, HSP has been reported to have a protective role against atrial fibrosis in several studies $(10,11)$; thirdly, abnormal expression of HSP70 has been detected in patients with pulmonary fibrosis and in rats with renal fibrosis $(12,13)$; and finally, by increasing the expression of Toll-like receptor 4, HSP70 interacts with vascular smooth muscle cells, which are the major producers of extracellular matrix proteins (14).

Geranylgeranylacetone (GGA), which is a drug used to treat gastric ulcers, has previously been observed to induce the expression of HSP70 in cultured gastric mucosal cells $(15,16)$. GGA-mediated upregulation of HSP70 has been demonstrated to prevent the majority of the important events that occur during fibrosis, including prevention of myofibroblast differentiation and epithelial-to-mesenchymal 
transition (EMT) (17,18). In addition, HSP70 has been reported to reduce apoptosis and EMT, which are important contributors to tubular cell injury in vitro and in vivo (12). Notably, a high dose of GGA has been observed to induce HSP72 in the rat liver $(19,20)$, and treatment with GGA may prevent liver damage through the induction of HSPs (21). Therefore, the present study hypothesized that GGA attenuates $\mathrm{CCl}_{4}$-induced fibrosis by upregulating the expression of HSP70.

The present study aimed to examine the hypothesis that GGA, an HSP70 inducer, may potentiate its biological effects against $\mathrm{CCl}_{4}$-induced liver injury via the upregulation of HSP70.

\section{Materials and methods}

Materials. The reagents used in the present study were obtained from the following sources: Geranylgeranylacetone (GGA) was purchased from Eisai China, Inc. (Shanghai, China). Anti-HSP70 was purchased from Cell Signaling Technology, Inc. (Beverly, MA, USA). $\mathrm{CCl}_{4}$ was purchased from Merck Millipore (Darmstadt, Germany). Transforming growth factor- $\beta 1$ (TGF- $\beta 1$; SC-146) antibody was purchased from Santa Cruz Biotechnology, Inc. (Dallas, TX, USA). $\alpha$-smooth muscle actin ( $\alpha$-SMA; A2547) and $\beta$-actin (A2228) antibodies were purchased from Sigma-Aldrich (St. Louis, MO, USA).

Animal models. A total of 24 male Sprague-Dawley rats, weighing between 180 and $220 \mathrm{~g}$, were housed under standard animal laboratory conditions, at a controlled temperature $\left(22 \pm 1^{\circ} \mathrm{C}\right)$, humidity $(65 \pm 5 \%)$ and $12 \mathrm{~h}$ light/dark cycles with free access to food and water, in a specific-pathogen-free-grade animal room at the Experimental Animal Center of Soochow University (Changzhou, China). All the rats were fasted for 2 days prior to the experiment. The rats were randomly divided into three groups: Group I (control group), group II ( $\mathrm{CCl}_{4}$ group), and group III $\left(\mathrm{CCl}_{4}+\mathrm{GGA}\right.$ group). The control rats were administered daily with sterile saline orally $(n=8)$. In the $\mathrm{CCl}_{4}$ model group, liver fibrosis was induced in the rats by intraperitoneal injection of $400 \mathrm{ml} / 1 \mathrm{CCl}_{4}$ salad oil solution, with a single dose of $3 \mu \mathrm{g} / \mathrm{g} / \mathrm{rat}$ twice a week $(\mathrm{n}=8)$. In the $\mathrm{CCl}_{4}$ model $+\mathrm{GGA}$ group, the rats received the same dose of $\mathrm{CCl}_{4}$ as group II, alongside a daily oral dose of an emulsion containing $400 \mathrm{mg} / \mathrm{kg}$ GGA, 4 weeks after modeling $(n=8)$. All the rats were sacrificed by injection with pentobarbital (65 mg/kg i.p.; Sigma-Aldrich) after 9 weeks, following which blood samples $(1 \mathrm{ml})$ and liver tissues were obtained. The animal experimental procedures were approved by the Animal Care and Use Committee of Soochow University and the Ethics Committee of the Third Affiliated Hospital of Soochow University (Changzhou, China).

Serological examination. At the end of the ninth week, blood samples were collected from the caudal vein of the rats and analyzed for levels of alanine aminotransferase (ALT), aspartate aminotransferase (AST), and total bilirubin (TB) using ALT/AST and TB kits (Nanjing Jiancheng Bioengineering Institue, Nanjing, China), according to the manufacturer's instructions. SPSS 13.0 softwared (SPSS, Inc., Chicago, IL, USA) was used for analysis and these measurements were used to compare the differences in liver function between each group.

Measurement of hepatic hydroxyproline content. Total hepatic hydroxyproline levels were determined in the hydrolysates of the liver samples. Briefly, $100 \mathrm{mg}$ wet liver samples were subjected to acid hydrolysis, and the quantity of hydroxyproline was determined using a Hydroxyproline Testing kit (cat. no. A030-2; Jiancheng, Nanjing, China), according to the manufacturer's instructions.

Histological examination. Liver biopsies from the rats were harvested and tissue fragments were fixed in Bouin's solution (Sigma-Aldrich) overnight, embedded in paraffin and stained with hematoxylin and eosin (HE) for general histopathological examination. Masson's trichrome staining and Sirius red staining were used to assess collagen levels. The red stained areas in the Sirius Red-stained sections were assessed using an image analyzer (Image-Pro Plus, Media Cybernetics, Inc., Rockville, MD, USA) for semiquantitative analysis. The percentage of Sirius Red staining was used to determine the differences in each group.

Immunofluorescence staining. Indirect immunofluorescence staining was performed, according to an established procedure (22). Briefly, $3 \mu \mathrm{m}$ cryosections were prepared using a Microm HM 440E (Thermo Fisher Scientific, Waltham, MA, USA) and blocked with $3 \%$ bovine serum albumin (BSA; Sigma-Aldrich) for $1 \mathrm{~h}$ at room temperature. Subsequently, the sections were incubated with primary antibodies against TGF- $\beta 1$ and $\alpha$-SMA in phosphate-buffered saline (PBS), supplemented with $3 \% \mathrm{BSA}$, overnight at $4^{\circ} \mathrm{C}$. The sections were then washed thoroughly in PBS, followed by incubation with tetramethylrhodamine-conjugated secondary antibody (SAB3700867; Sigma-Aldrich), at a dilution of 1:200, in PBS supplemented with $3 \%$ BSA, in the dark for $1 \mathrm{~h}$. Following subsequent washing with PBS, the sections on slides were visualized using a Nikon Eclipse 80i Epi-fluorescence microscope equipped with a digital camera (DS-Ri1; Nikon Corporation, Tokyo, Japan).

Western blot analysis. The total proteins were extracted using radioimmunoprecipitation buffer containing a protease inhibitor cocktail (Sigma-Aldrich). The protein concentrations were determined using a bichinchoninic acid method (Beyotime Institute of Biotechnology, Haimen, China). Equal quantities of protein $(50 \mu \mathrm{g})$ were loaded onto each lane of a polyacrylamide gel, separated by $10 \%$ SDS-PAGE (EMD Millipore, Billerica, MA, USA) and transferred to nitrocellulose membranes (EMD Millipore). The membranes were blocked with 5\% skimmed milk in Tris-buffered saline $(\mathrm{pH} 7.6)$ at room temperature, and were then incubated overnight at $4^{\circ} \mathrm{C}$ with the following primary antibodies: Anti-HSP70 (4008; 1:1,000), anti-TGF- $\beta 1$ (1:200), anti- $\alpha$-SMA (1:200) and anti- $\beta$-actin (1:500). Following further incubation with the corresponding secondary antibody, immune complexes (7074) were detected using enhanced chemiluminescence western blotting reagents (Sinopharm Chemical Reagent, Shanghai, China). The detected proteins were normalized to $\beta$-actin or the respective total protein, as appropriate. 


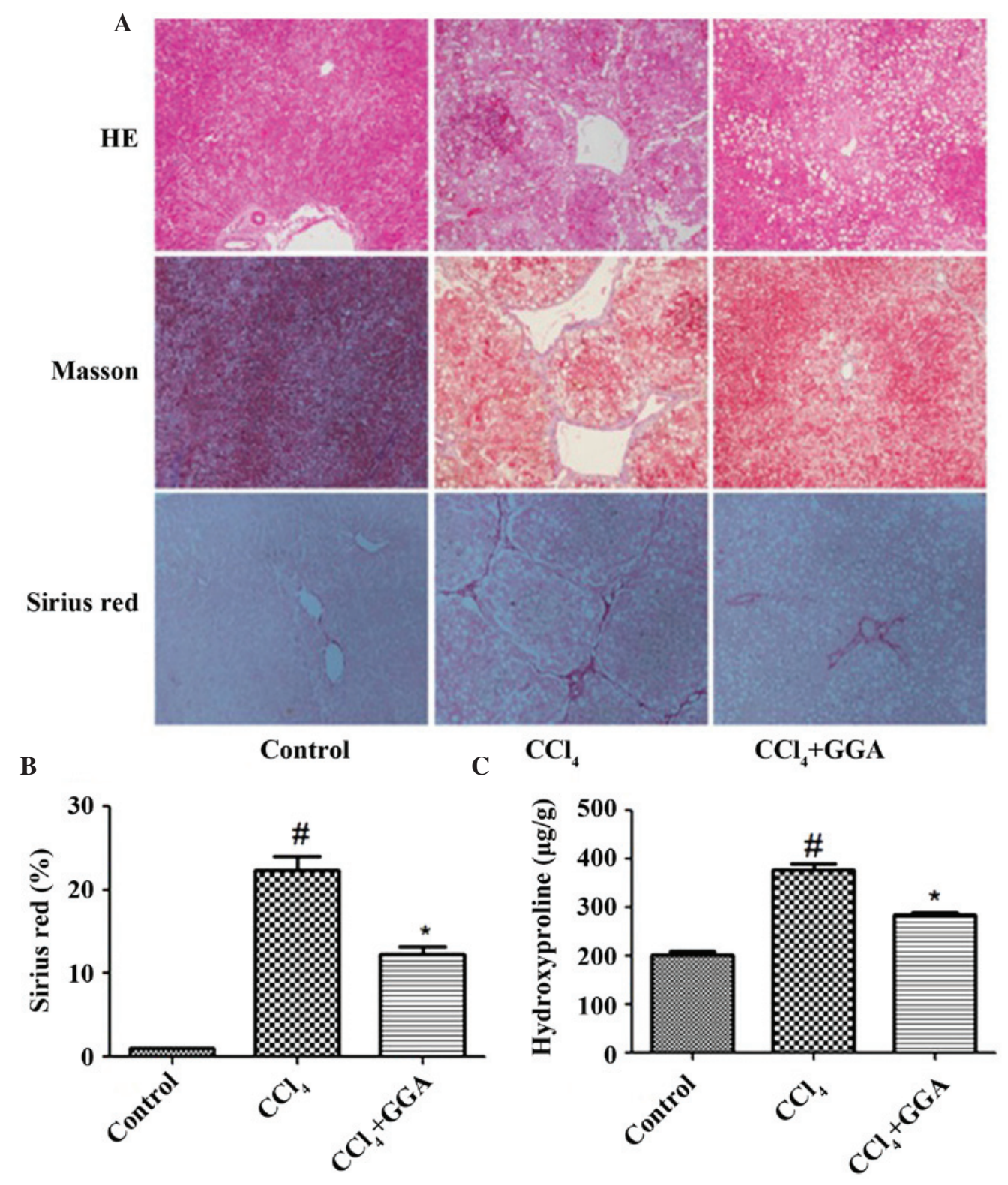

Figure 1. GGA attenuated $\mathrm{CCl}_{4}$-induced hepatic fibrosis in rats. (A) $\mathrm{CCl}_{4}$ was used to construct a hepatic fibrosis model, to evaluate the therapeutic effect of GGA. Liver sections were stained with HE, Masson's reagent and Sirius Red for histological examination to determine hepatic morphology, structural changes and ECM content in the liver tissue of each group (magnification, x200). (B and C) Levels of ECM were determined by quantitative estimation of the red-stained areas in the Sirius Red-stained sections, and of hydroxyproline content. ${ }^{\prime \prime} \mathrm{P}<0.05$, compared with the control group; "P $<0.05$, compared with the $\mathrm{CCl}_{4}$ group. GGA, geranylgeranylacetone; $\mathrm{CCl}_{4}$, carbon tetrachloride; $\mathrm{HE}$, hematoxylin and eosin; ECM, extracellular matrix.

Statistical analysis. Statistically significant differences between the control and treatment groups were detected using a simple analysis of variance, followed by Dunnett's multiple comparison tests. SPSS 13.0 was used for statistical analysis. $\mathrm{P} \leq 0.05$ was considered to indicate a statistically significant difference.

\section{Results}

Effects of GGA on hepatic fibrosis in $\mathrm{CCl}_{4}$-induced rats. As shown in Fig. 1, the rats in the $\mathrm{CCl}_{4}$-induced group exhibited extensive fibrotic deposition, which was demonstrated by HE, Masson's trichrome and Sirius red staining. In addition, quantitative estimation of hydroxyproline content indicated that the hydroxyproline content was significantly higher in the $\mathrm{CCl}_{4}$-induced group, compared with the control group $(\mathrm{P}<0.05)$. However, treatment with GGA resulted in significant decreases in fibrotic deposition and the content of hydroxyproline, compared with the $\mathrm{CCl}_{4}$-induced group $(\mathrm{P}<0.05)$.

Effects of GGA on liver function in $\mathrm{CCl}_{4}$-induced rats. As shown in Fig. 2, the ALT, AST, and TB levels were markedly increased in the $\mathrm{CCl}_{4}$-treated rats, compared with the control group. However, the GGA-treated $\mathrm{CCl}_{4}$ rats exhibited marked decreases in ALT, AST and TB levels, compared with the $\mathrm{CCl}_{4}$-treated rats.

Effects of GGA on the expression of HSP70 in $\mathrm{CCl}_{4}$-induced rats. To determine the role of HSP70 in the protective effects of GGA against $\mathrm{CCl}_{4}$-induced hepatic damage, the expression 
A

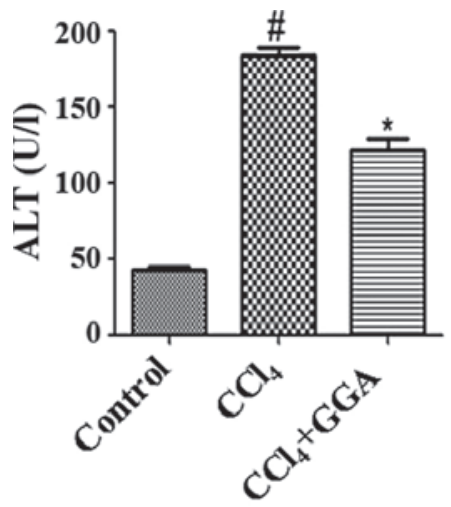

B

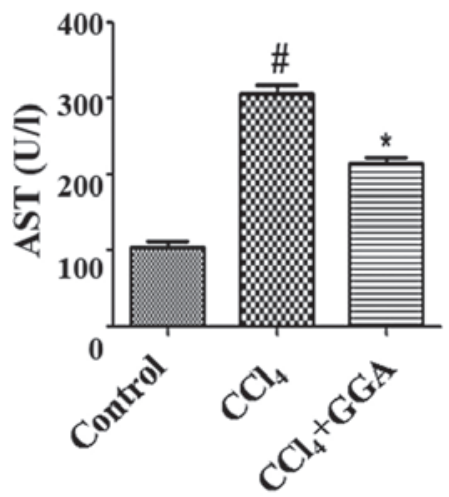

C

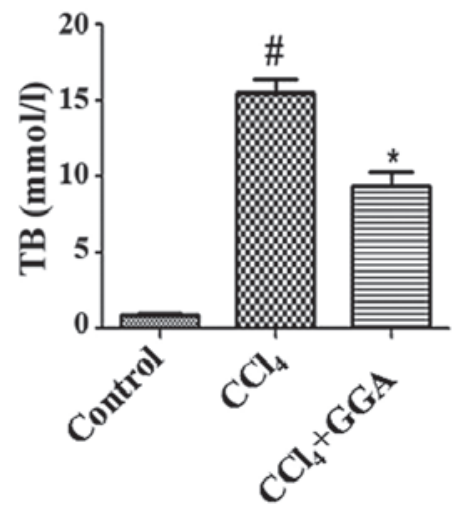

Figure 2. GGA improves liver function in $\mathrm{CCl}_{4}$-induced rats. The levels of (A) ALT, (B) AST, and (C) TB were examined to assess hepatic function. ${ }^{\#} \mathrm{P}<0.05$, compared with the control group; ${ }^{*} \mathrm{P}<0.05$, compared with the $\mathrm{CCl}_{4}$ group. GGA, geranylgeranylacetone; $\mathrm{CCl}_{4}$, carbon tetrachloride; ALT, alanine aminotransferase; AST, aspartate aminotransferase; TB, total bilirubin.

levels of HSP70 were examined using western blotting. Treatment with $\mathrm{CCl}_{4}$ resulted in a significant increase in the protein expression levels of HSP70 in the liver, compared with the control group. Treatment with GGA was found to further enhance $\mathrm{CCl}_{4}$-induced expression of HSP70 in the liver (Fig. 3). These results indicated that GGA induced the expression of HSP70, which may be associated with the protective effects of GGA against $\mathrm{CCl}_{4}$-induced hepatic fibrosis.

Effects of GGA on the expression levels of profibrogenic proteins in $\mathrm{CCl}_{4}$-induced rats. Immunofluorescence staining and western blotting were used to detect the expression levels of $\alpha$-SMA and TGF- $\beta 1$ in the liver tissues of the rats. $\alpha$-SMA and TGF- $\beta 1$ have been reported to be involved with the development of hepatic fibrosis $(23,24)$. The expression levels
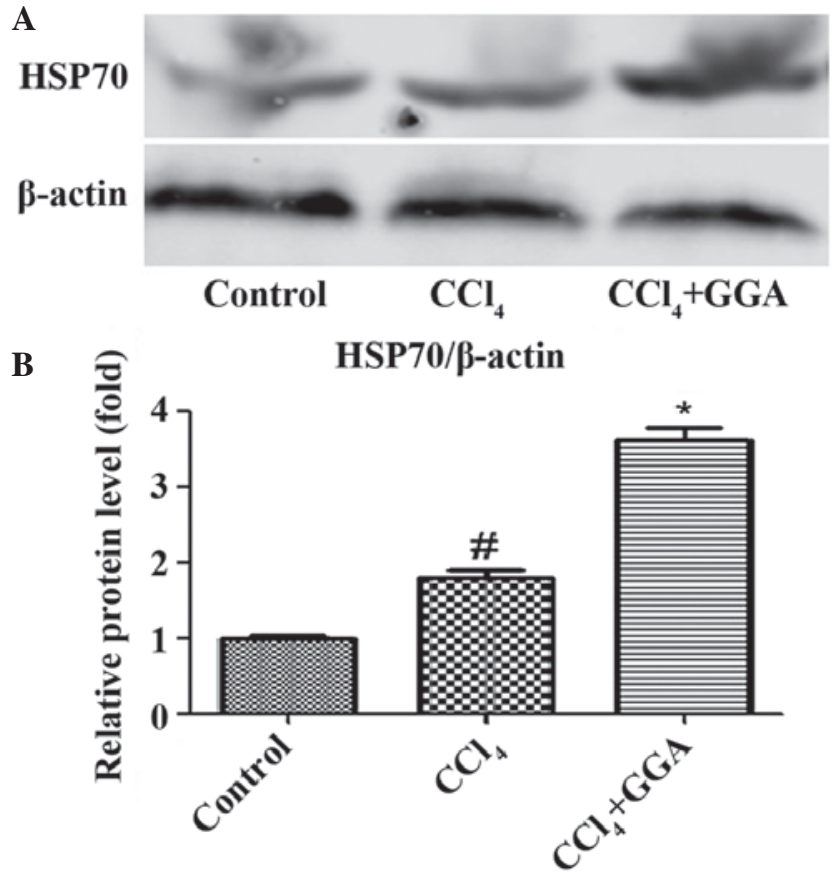

Figure 3. GGA further increases the expression of $\mathrm{HSP} 70$ in $\mathrm{CCl}_{4}$-induced rats. (A) Protein samples $(50 \mu \mathrm{g})$ were loaded for western blot analysis, in order to determine the expression levels of HSP70. Similar results were obtained from three repeated experiments. (B) Quantitative analysis was performed for HSP70 using a densitometer. Data are presented as the mean \pm standard deviation. ${ }^{\#} \mathrm{P}<0.05$, compared with the control group; ${ }^{*} \mathrm{P}<0.05$, compared with the $\mathrm{CCl}_{4}$ GGA group. GGA, geranylgeranylacetone; $\mathrm{CCl}_{4}$, carbon tetrachloride; HSP70, heat shock protein 70 .

of $\alpha$-SMA and TGF- $\beta 1$ were low in the liver tissue of the control rats; however, a significant increase in the expression of $\alpha$-SMA and TGF- $\beta 1$ was observed with the development of $\mathrm{CCl}_{4}$-induced hepatic fibrosis. Treatment with GGA was found to markedly reduce the expression levels of hepatic fibrosis-associated proteins (Fig. 4).

\section{Discussion}

In previous decades, there have been significant advances in understanding of the cellular and molecular mechanisms underlying liver fibrogenesis. Hepatic fibrosis is the common response to chronic liver injury, which may result in slow, progressive damage to liver function and disrupt the structure of liver tissue $(1,2)$. Hepatic fibrosis is also a reversible wound-healing response to either acute or chronic cellular injury, which reflects the balance between liver repair and scar formation (25). Identifying the mechanisms underlying liver fibrogenesis is important for the development of targeted therapies to reverse the fibrotic response and improve the prognosis of patients with chronic liver disease. HSP70 is involved in several cellular processes and exerts a wide range of functions. HSP70 is involved in the prevention of protein aggregation through redirecting unfolded or misfolded proteins to the proteasomal degradation system (26). Previous studies have demonstrated that HSP70 has a protective role against fibrogenesis $(25,27-29)$. However, further investigation is required to clearly determine its roles in hepatic fibrogenesis (30). 


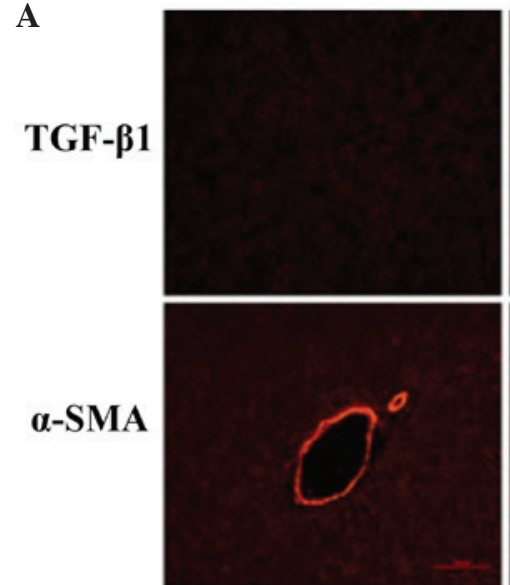

Control
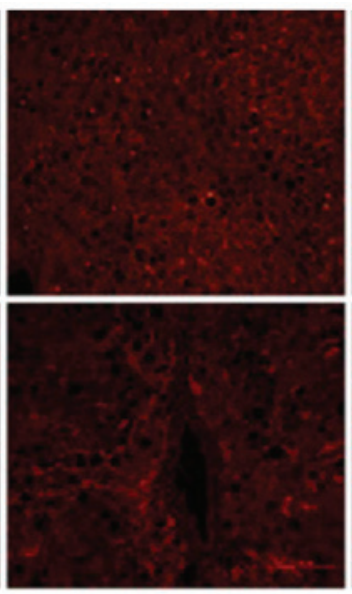

$\mathrm{CCl}_{4}$
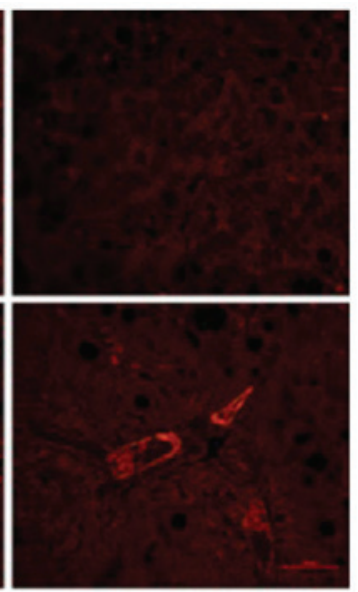

$\mathrm{CCl}_{4}+\mathbf{G G A}$

B
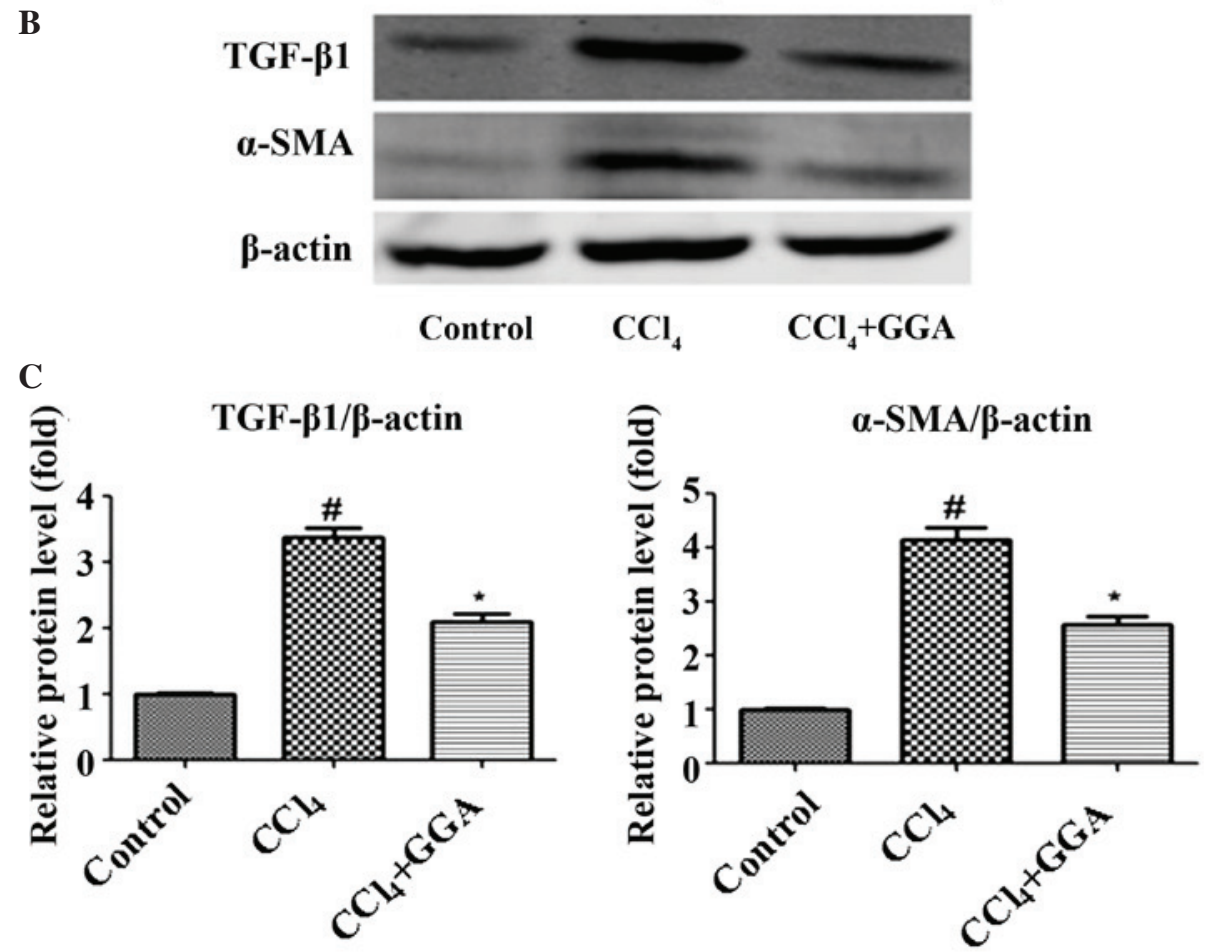

Figure 4. GGA attenuates profibrogenic protein expression in $\mathrm{CCl}_{4}$-induced rats. (A) Immunofluorescence was used to examine the expression levels of TGF- $\beta 1$ and $\alpha$-SMA in the liver tissues (magnification, x200). (B) Protein samples $(50 \mu \mathrm{g}$ ) were loaded for western blot analysis of TGF- $\beta 1$ and $\alpha$-SMA. Similar results were obtained from three repeated experiments. (C) Quantitative analysis was performed for TGF- $\beta 1$ and $\alpha$-SMA using a densitometer. Data are presented as the mean \pm standard deviation. ${ }^{"} \mathrm{P}<0.05$, compared with the control group; ${ }^{*} \mathrm{P}<0.05$ compared with the $\mathrm{CCl}_{4}$ group. $\mathrm{GGA}$, geranylgeranylacetone; $\mathrm{CCl}$, carbon tetrachloride; TGF- $\beta 1$, transforming growth factor- $\beta 1, \alpha$-SMA, $\alpha$-smooth muscle actin.

The results of the present study demonstrated that $\mathrm{CCl}_{4}$ significantly increased the expression levels of HSP70 in the liver, compared with the control group. Treatment with GGA, an inducer of HSP70, further enhanced the $\mathrm{CCl}_{4}$-induced expression of HSP70 in the liver, compared with the $\mathrm{CCl}_{4}$-induced group. Notably, GGA administration attenuated $\mathrm{CCl}_{4}$-induced hepatic fibrosis, as evidenced by the results of the HE, Masson's trichrome and Sirius red staining, and the quantitative estimation of hydroxyproline content. In addition, GGA improved the liver function, compared with the $\mathrm{CCl}_{4}$-induced rats. As indicators of hepatic fibrosis, the expression levels of TGF- $\beta 1$ and $\alpha$-SMA in the GGA-treated $\mathrm{CCl}_{4}$ group were found to be markedly lower than those in the $\mathrm{CCl}_{4}$-induced rats. These results indicated that GGA attenuated hepatic fibrosis through increasing the expression of HSP70.

In conclusion, the identification of therapeutic applications to prevent the progression of liver fibrosis is clinically beneficial. The present study is the first, to the best of our knowledge, to demonstrate that daily oral administration of GGA may induce the expression of $\mathrm{HSP} 70$ in $\mathrm{CCl}_{4}$-treated rat liver. Furthermore, HSP70 upregulation may be used as a novel therapeutic approach for the prevention of hepatic fibrosis.

\section{Acknowledgements}

The authors would like to thank Dr Bin Wang (Division of Nephrology, Huashan Hospital and Institute of Nephrology, 
Fudan University, Shanghai, China) for technical assistance and helpful discussions.

\section{References}

1. Bellaye PS, Burgy O, Causse S, Garrido C and Bonniaud P: Heat shock proteins in fibrosis and wound healing: Good or evil? Pharmacol Ther 143: 119-132, 2014.

2. Booz GW, Carl LL and Baker KM: Amplification of angiotensin II signaling in cardiac myocytes by adenovirus-mediated overexpression of the AT1 receptor: Ann NY Acad Sci 874: 20-26, 1999.

3. Bukau B and Horwich AL: The Hsp70 and Hsp60 chaperone machines. Cell 92: 351-366, 1998.

4. Friedman SL: Molecular regulation of hepatic fibrosis, an integrated cellular response to tissue injury. J Biol Chem 275: 2247-2250, 2000.

5. Friedman SL: Hepatic stellate cells: Protean, multifunctional, and enigmatic cells of the liver. Physiol Rev 88: 125-172, 2008.

6. Shiber A and Ravid T: Chaperoning proteins for destruction: Diverse roles of Hsp70 chaperones and their co-chaperones in targeting misfolded proteins to the proteasome. Biomolecules 4: 704-724, 2014.

7. Fudaba Y, Ohdan H, Tashiro H, Mizunuma K, Ito H, Fukuda Y, Dohi K and Asahara T: High dose of geranylgeranylacetone accumulate HSP72 mRNA in rat liver. Transplant Proc 33: 978, 2001.

8. Reus IS, Bando I, Andres D and Cascales M: Relationship between expression of HSP70 and metallothionein and oxidative stress during mercury chloride induced acute liver injury in rats. J Biochem Mol Toxicol 17: 161-168, 2003.

9. Fudaba Y, Tashiro H, Ohdan H, Miyata Y, Shibata S, Shintaku S, Nishihara M, Asahara T, Ito H, Fukuda Y and Dohi K: Efficacy of HSP72 induction in rat liver by orally administered geranylgeranylacetone. Transpl Int (13 Suppl 1): S278-S281, 2000.

10. Fujibayashi T, Hashimoto N, Jijiwa M, Hasegawa Y, Kojima T and Ishiguro N: Protective effect of geranylgeranylacetone, an inducer of heat shock protein 70, against drug-induced lung injury/fibrosis in an animal model. BMC Pulm Med 9: 45, 2009.

11. Goette A, Staack T, Röcken C, Arndt M, Geller JC, Huth C, Ansorge S, Klein HU and Lendeckel U: Increased expression of extracellular signal-regulated kinase and angiotensin-converting enzyme in human atria during atrial fibrillation. J Am Coll Cardiol 35: 1669-1677, 2000.

12. González-Ramos M, Calleros L, López-Ongil S, Raoch V, Griera M, Rodríguez-Puyol M, de Frutos S and Rodríguez-Puyol D: HSP70 increases extracellular matrix production by human vascular smooth muscle through TGF- $\beta 1$ up-regulation. Int J Biochem Cell Biol 45: 232-242, 2013.

13. Kahloon RA, Xue J, Bhargava A, Csizmadia E, Otterbein L, Kass DJ, Bon J, Soejima M, Levesque MC, Lindell KO, et al: Patients with idiopathic pulmonary fibrosis with antibodies to heat shock protein 70 have poor prognoses. Am J Respir Crit Care Med 187: 768-775, 2013.

14. Kanemura H, Kusumoto K, Miyake H, Tashiro S, Rokutan K and Shimada M: Geranylgeranylacetone prevents acute liver damage after massive hepatectomy in rats through suppression of a CXC chemokine GRO1 and induction of heat shock proteins. J Gastrointest Surg 13: 66-73, 2009.
15. Lee UE and Friedman SL: Mechanisms of hepatic fibrogenesis. Best Pract Res Clin Gastroenterol 25: 195-206, 2011.

16. Luo FC, Zhao L, Deng J, Liang M, Zeng XS, Liu H and Bai J: Geranylgeranylacetone protects against morphine-induced hepatic and renal damage in mice. Mol Med Rep 7: 694-700, 2013.

17. Mao H, Li Z, Zhou Y, Li Z, Zhuang S, An X, Zhang B, Chen W, Nie J, Wang Z, et al: HSP72 attenuates renal tubular cell apoptosis and interstitial fibrosis in obstructive nephropathy. Am J Physiol Renal Physiol 295: F202-F214, 2008.

18. Myung SJ, Yoon JH, Kim BH, Lee JH, Jung EU and Lee HS: Heat shock protein 90 inhibitor induces apoptosis and attenuates activation of hepatic stellate cells. J Pharmacol Exp Ther 330: 276-282, 2009.

19. Ooie T, Takahashi N, Saikawa T, Nawata T, Arikawa M, Yamanaka K, Hara M, Shimada T and Sakata T: Single oral dose of geranylgeranylacetone induces heat-shock protein 72 and renders protection against ischemia/reperfusion injury in rat heart. Circulation 104: 1837-1843, 2001.

20. Routsias JG and Tzioufas AG: The role of chaperone proteins in autoimmunity. Ann NY Acad Sci 1088: 52-64, 2006.

21. Takahashi N, Kume O, Wakisaka O, Fukunaga N, Teshima Y, Hara M and Saikawa T: Novel strategy to prevent atrial fibrosis and fibrillation. Circ J 76: 2318-2326, 2012.

22. Chen J: Chloroquine improved carbon tetrachloride-induced liver fibrosis through its inhibition of the activation of hepatic stellate cells: Role of autophagy. Biol Pharm Bull 37: 1505-1509, 2014.

23. Nouchi T, Tanaka Y, Tsukada T, Sato C and Marumo F: Appearance of alpha-smooth-muscle-actin-positive cells in hepatic fibrosis. Liver 11: 100-105, 1991.

24. Hellerbrand C, Stefanovic B, Giordano F, Burchardt ER and Brenner DA. The role of TGFbetal in initiating hepatic stellate cell activation in vivo. J Hepatol 30: 77-87, 1999.

25. Tanaka K, Tanaka Y, Namba T, Azuma A and Mizushima T: Heat shock protein 70 protects against bleomycin-induced pulmonary fibrosis in mice. Biochem Pharmacol 80:920-931, 2010.

26. Shin Y, Klucken J, Patterson C, Hyman BT and McLean PJ: The co-chaperone carboxyl terminus of Hsp70-interacting protein (CHIP) mediates alpha-synuclein degradation decisions between proteasomal and lysosomal pathways. J Biol Chem 280: 23727-23734, 2005.

27. Tsuruma T, Yagihashi A, Koide S, Araya J, Tarumi K, Watanabe $\mathrm{N}$ and Hirata K: Geranylgeranylacetone induces heat shock protein-73 in rat small intestine. Transplant Proc 31: 572-573, 1999.

28. Wakisaka O, Takahashi N, Shinohara T, Ooie T, Nakagawa M, Yonemochi H, Hara M, Shimada T, Saikawa T and Yoshimatsu H: Hyperthermia treatment prevents angiotensin II-mediated atrial fibrosis and fibrillation via induction of heat-shock protein 72 . J Mol Cell Cardiol 43: 616-626, 2007.

29. Wu C: Heat shock transcription factors: Structure and regulation. Annu Rev Cell Dev Biol 11: 441-469, 1995.

30. Wynn TA: Common and unique mechanisms regulate fibrosis in various fibroproliferative diseases. J Clin Invest 117: 524-529, 2007. 